\title{
Cui bono? De la polémique sur les revenus au budget global
}

\author{
Jürg Schlup \\ Dr méd., président de la FMH
}

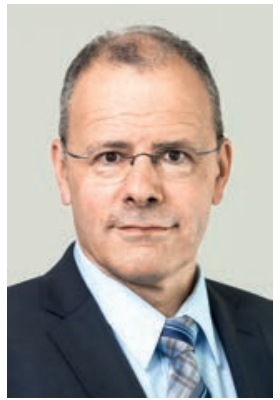

L’année 2018 se termine comme elle a débuté, par une polémique sur les revenus des médecins lancée par la politique. En janvier déjà, le conseiller d'Etat genevois responsable de la santé "estimait» - publiquement et sans citer aucune source - que les revenus des chirurgiens étaient "proches du million» [1]. Des propos repris le même jour au téléjournal par le chef du Département de l'intérieur (DFI): "Cela veut dire jusqu'à 80000 ou 90000 francs par mois payés par les primes» [2]. Ce qui est impossible: pour cela, un médecin devrait travailler tous les jours 23 heures en ambulatoire.

Sur question du Parlement, le DFI a précisé le 16 mai 2018, toujours sans citer de source, qu'«il n'y a [...] pas de transparence dans ce domaine» car "en ce qui concerne les revenus soumis à l'AVS des médecins exerçant en cabinet privé, il n'existe pas d'enquêtes systématiques autres que celles mandatées par la FMH» [3]. Ces explications surprennent d'autant plus que l'Office fédéral de la statistique (OFS) a publié le 10 avril 2018 l'évaluation 2017 des données fournies par 7000 cabinets, qui fait état d'un revenu de 155000 francs, dont 138000 issus des primes $[4,5]$. Pourquoi avoir omis ces chiffres dans la réponse du DFI?

\section{Dans quel but mettre en avant les 101 revenus les plus élevés d'une étude portant sur 21347 médecins?}

Fin octobre à nouveau, l'Office fédéral de la santé publique (OFSP) lance une fausse image, en commentant son étude sur les "revenus des médecins en Suisse», qui ne couvre pas «90\%» du corps médical comme affirmé [6] mais exclut le tiers des médecins aux revenus les plus bas. Dans sa communication, l'OFSP généralise à partir d'une petite minorité [6]: une lecture attentive de l'étude [7] montre que parmi toutes les disciplines et 21347 médecins, seuls deux sous-groupes présentent un revenu médian aussi élevé que celui cité en introduction au communiqué de presse de l'OFSP, à savoir 26 neurochirurgiens sur 118 et 75 gastroentérologues sur 233 [6]. Les auteurs de l'étude précisent d'ailleurs que les données sont à interpréter avec précaution en raison du faible nombre de cas [7], ce que l'OFSP ne mentionne pas dans son communiqué.

On peut se demander dans quel but mettre en avant le 0,5\% de revenus médians les plus élevés parmi les 21347 médecins étudiés, sans préciser la taille des groupes concernés. Cela rend toute discussion fondée très difficile, d'autant plus que l'OFSP a mis son étude de 128 pages à notre disposition seulement deux heures avant d'en informer les médias, alors qu'elle était disponible depuis plusieurs semaines déjà.

A qui profite cette désinformation sur les salaires des médecins? Avant tout à ceux qui veulent atteindre une

La désinformation profite avant tout à ceux qui poursuivent une majorité politique en faveur de plafonds budgétaires.

majorité politique en faveur de plafonds budgétaires, comme le préconise le premier volet de mesures du DFI visant à maîtriser les coûts de la santé, actuellement en consultation. Il doit contraindre les partenaires tarifaires à piloter les coûts selon les objectifs fixés et avec une compétence subsidiaire de la Confédération [8]. Dans un premier temps, il est prévu de "définir ce qu'est une hausse des coûts ‘acceptable〉, c.-̀̀-d. justifiée» [9] soit un budget global à ne pas dépasser, la sanction étant "une adaptation des tarifs" [9].

Certains ne l'ont peut-être pas réalisé: il s'agit là d'un projet de loi visant à introduire un budget global (ou un tarif dégressif). Plus les revenus des médecins sont décriés, plus les chances de ce projet augmentent. L'article à la page 1724 [10] illustre clairement les conséquences négatives d'une budgétisation, sur la rémunération des médecins certes, mais avant tout sur les soins médicaux. La FMH va continuer à se battre pour l'autonomie tarifaire et contre les budgets globaux, synonymes de médecine à deux vitesses et de rationnement. 


\section{Références}

1 RTS 1, La Matinale, 29.1.2018, 6h30, L'invité de Romain Clivaz (vidéo) - Mauro Poggia, conseiller d'Etat genevois; URL: https://www.rts. $\mathrm{ch} /$ play/radio/la-matinale/video/linvite-de-romain-clivaz-video-mauro-poggia-conseiller-detat-genevois?id=9287942\&s tation=a9e7621504c6959e35c3ecbe7f6bed0446cdf8da (à partir de 3:50)

2 RTS, 19h30, 29.1.2018, URL: https://www.rts.ch/play/tv/19h30 signe/video/19h30-signe?id=9290070 (à partir de 7:14)

3 Interpellation parlementaire 18.3015. CN Olivier Feller du 26 février 2018 «De quel chapeau le président de la Confédération Alain Berset a-t-il sorti les statistiques sur les revenus des médecins?»; URL: https://www.parlament.ch/fr/ratsbetrieb/suche-curia-vista/ geschaeft?AffairId=20183015

4 Statistique des cabinets médicaux et centres ambulatoires (MAS), Office fédéral de la statistique (OFS), 10.4.2018; URL: https://www. bfs.admin.ch/bfs/fr/home/statistiques/sante/systeme-sante/cabinets-medicaux.\%C2\%ADassetdetail.4924760.html

5 Schlup J. Des chiffres pertinents grâce à la collaboration des médecins. Bull Med Suisses. 2018;99(43):1479; URL: https://bullmed.ch/ article/doi/saez.2018.17283

6 Communiqué de presse de l'OFSP du 29 octobre 2018. Revenus des médecins en Suisse: une nouvelle étude améliore la transparence; URL: https://www.admin.ch/gov/fr/accueil/documentation/communiques.msg-id-72699.html

7 Künzi K, Morger M. Berne, 28 août 2018. Einkommen, OKP-Leistun gen und Beschäftigungssituation der Ärzteschaft 2009-2014. Analyse verknüpfter Datensätze: Medizinalberuferegister BAG, AHVDaten der Zentralen Ausgleichsstelle, Krankenversicherungsdaten SASIS. Schlussbericht. Im Auftrag des Bundesamts für Gesundheit BAG. Direktionsbereich Gesundheitspolitik (en allemand).
8 Acte modificateur relatif à la révision partielle de la loi fédérale sur l'assurance-maladie: Mesures visant à freiner la hausse des coûts $-1^{\text {er }}$ volet (PDF, $\left.361 \mathrm{~KB}, 14.9 .2018\right) ;$ URL: https://www.bag.admin.ch/ $\mathrm{dam} / \mathrm{bag} / \mathrm{fr} /$ dokumente/kuv-leistungen/Laufende\%20Revisionsprojekte/kvg-revision-kostendaempfung-paket-1/aenderungserlass-zur-teilrevision-des-bundesgesetzes-ueber-diekrankenversicherung-massnahmen-zur-kostendaempfung-paket-1.

pdf.download.pdf/\%C3\%84nderungserlass_Kostend\%C3\%A4mpfung\%20Paket\%201_FR.pdf

9 Rapport explicatif concernant la révision partielle de la loi fédérale sur l'assurance-maladie: Mesures visant à freiner la hausse des coûts $-1^{\text {er }}$ volet (PDF, 1 MB, 14.9.2018); URL: https://www.bag.admin $\mathrm{ch} / \mathrm{dam} / \mathrm{bag} / \mathrm{fr} /$ dokumente/kuv-leistungen/Laufende\%20Revisionsprojekte/kvg-revision-kostendaempfung-paket-1/erlaeutern der-bericht-zur-teilrevision-des-bundesgesetzes-ueber-die-krankenversicherung-massnahmen-zur-kostendaempfung-paket-1. pdf.download.pdf/Erl\%C3\%A4uternder\%2OBericht_ Kostend\%C3\%A4mpfung\%2OPaket\%201 FR.pdf

10 Wille N, Schlup J. L'impact des budgets sur les soins ambulatoires. Bull Med Suisses. 2018;99(49):1724-25; https://bullmed.ch/fr/ article/doi/bms.2018.17385/ 